\title{
Russell y Wittgenstein: sobre contradicciones y paradojas
}

\author{
Alejandro Tomasini
}

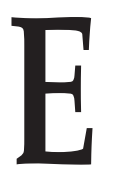

\section{1 problema}

Pienso que sería útil comenzar este ensayo con una aclaración puramente lingüística concerniente a algunos términos clave. Los conceptos de contradicción y de paradoja pertenecen a una determinada familia de conceptos respecto de los cuales no es inusual creer que todos ellos significan más o menos lo mismo y que son intercambiables salva veritate. Esto, hay que decirlo de inmediato, no es el caso. Por lo pronto, es importante distinguir al menos cuatro nociones, a saber, las de paradoja, contradicción, antinomia y aporía. (Dejo fuera la de paralogismo, que es una noción exclusivamente kantiana.) El objetivo de este preámbulo aclaratorio es el de ponernos en la vía apropiada para enfocar del modo correcto nuestro problema. Consideremos, pues, en primer lugar, la noción de antinomia. Una antinomia se compone de dos argumentos, ambos a primera vista válidos y tales que sus conclusiones son proposiciones igualmente plausibles, pero mutuamente excluyentes; en el caso de una antinomia ambas conclusiones parecen haber sido demostradas. Por otra parte, una aporía tiene la misma estructura que una antinomia, sólo que en ella las conclusiones parecen ser igualmente implausibles. En este segundo caso, también las conclusiones parecen haber sido probadas por lo que prima facie son argumentos lógicamente correctos. Ahora bien, los conceptos de antinomia y de aporía son irrelevantes para el problema que aquí nos ocupa, sobre todo porque involucran a la noción de verdad de un modo que no es meramente formal. Para entender mejor esto consideremos la noción de contradicción. Tal vez la forma más sencilla de definir contradicción sea decir que es una instancia o ejemplificación de la fórmula ' $\varphi \mathbf{v} \sim \varphi$ '. En otras palabras, una contradicción es la conjunción de una proposición y de su negación. Pero debería observarse que las dos caracterizaciones no necesariamente coinciden o al menos no lo hacen de manera 
obvia. Considérese, por ejemplo, a la oración 'Todos los hombres son mortales'. No está claro cuál sea la negación de esto en el lenguaje natural. En el simbolismo lógico, la conjunción de esta oración y su negación da como resultado ' $(x)(\mathrm{H} x \rightarrow \mathrm{M} x) \&(\exists x)(\mathrm{H} x \& \sim \mathrm{M} x)^{\prime}$, que no es una instancia de sustitución de ' $(\varphi \vee \sim \varphi)$ '. De ahí que las proposiciones contradictorias no necesariamente lo sean en virtud de la sintaxis o de su estructura interna, sino que también el significado es relevante. Queda como una cuestión abierta la de si en última instancia todas las contradicciones son o no casos particulares de la fórmula arriba mencionada. Por el momento, sin embargo, nos contentaremos con definir contradicción como la conjunción de dos proposiciones tales que, versando sobre lo mismo, una es verdadera si y solamente si la otra es falsa. Ahora bien, hay que decir que, en contraste con una creencia ampliamente difundida, una paradoja no es simplemente una contradicción; en todo caso, es un par de contradicciones a las que se llega mediante razonamientos lógicamente impecables. A diferencia de lo que acontece con las antinomias, con las paradojas claramente "vemos" que las contradicciones se siguen lógicamente en cada caso posible de sus respectivas premisas, es decir, tanto si se acepta una tesis como si se acepta su negación. Una paradoja es algo que hay que evitar porque desemboca en contradicciones (entre premisas y conclusiones aceptables de argumentos aparentemente válidos). Pero cabe entonces preguntar: ¿¿uál es el problema con las contradicciones? ¿Por qué habríamos de temerles? A grandes rasgos, pienso que podemos dar dos clases de respuestas a estas preguntas:

a) Si la pregunta no se la hacemos a lógicos, a matemáticos o a filósofos, la respuesta probablemente será que se trata de expresiones inútiles, de expresiones que quizá en algún sentido nos desconciertan, que probablemente no habría que hacerles mucho caso, etcétera. Llamaré a esta clase de respuesta "sensata", y la consideraré en detalle en la cuarta parte de este trabajo.

b) La respuesta del lógico, la cual consistirá en señalar que si en verdad no fuera posible bloquear la gestación de contradicciones, entonces estaríamos en el grave riesgo de vernos facultados para deducir absolutamente lo que nos venga en gana, dando así al traste con nuestros mecanismos normales de inferencias. Ilustremos esto. Supóngase que estamos trabajando en un sistema formal (estamos aquí describiendo lo que sucede en el lenguaje objeto). Es bien sabido que, de acuerdo con la lógica, si en una línea $(n)$ de mi cadena de deducciones tengo una proposición $\varphi$ y en una línea $(n+1)$ tengo $\sim \varphi$, entonces en una línea $(n+2)$ puedo escribir $(\varphi \vee \psi)$ y, finalmente, en una línea $(n+3)$ puedo obtener $\psi$. 
Gráficamente:

(n) $\varphi$

$(\mathrm{n}+1) \sim \varphi$

$(\mathrm{n}+2)(\varphi \vee \psi) \quad($ de $(\mathrm{n}) \mathrm{y}$ def. de ' $\mathrm{v}$ ')

$(n+3) \psi \quad(\operatorname{de}(n+1),(n+2) y$ def. de 'v')

El problema, evidentemente, es que ' $\psi$ ' puede ser cualquier proposición. Así, pues, si no logramos imponer ciertas restricciones a nuestros razonamientos, sería posible que se nos obligara a admitir absolutamente cualquier cosa. Dado que no queremos eso, debemos tratar a toda costa de evitar incurrir o caer en contradicciones, y por ende en paradojas. Si fuéramos incapaces de lograrlo, podríamos entonces quizá vernos en la situación en la que Russell puso a Frege en 1900, quien, después de haber mostrado que la aritmética y algunas otras ramas de la matemática pueden explicarse en términos puramente lógicos y que sus proposiciones pueden deducirse de unas cuantas proposiciones lógicas, fue informado por Russell que su sistema, dada la forma como él había empleado ciertos conceptos (como el de clase), contenía una paradoja. Por lo tanto, era posible deducir en dicho sistema, por ejemplo, que $4=5$ o, más en general, lo que uno quisiera. Frege había usado la noción de clase (conjunto) y también un axioma irrestricto de comprehensión. Lo que esto quiere decir es que, de acuerdo con él, cualquier función proposicional determina un conjunto, a saber, el conjunto de los objetos que satisfacen la función en cuestión (el cual puede, naturalmente, ser el conjunto vacío). Russell invitó entonces a Frege a que considerara el caso de la función ' $x$ es miembro de sí mismo'. De acuerdo con Frege, debería haber un conjunto constituido por elementos que satisficieran esta función y, claro está, que fueran diferentes del conjunto en cuestión. Esto es lo que se quiere decir mediante 'miembro de sí mismo' o 'no miembro de sí mismo'. Claramente, hay conjuntos que no son miembros de sí mismos. El conjunto de todos los elefantes, por ejemplo, no es un elefante. Pero también hay conjuntos que sí son miembros de sí mismos. Por ejemplo, el conjunto de las cosas que no son leones no es un león. Ahora bien, consideremos el conjunto de todos los conjuntos que no son miembros de sí mismos y preguntémonos: ¿es él un miembro de sí mismo? Hay sólo dos respuestas posibles: o es un miembro de sí mismo o no lo es. Si es un miembro de sí mismo, entonces, puesto que pertenece al conjunto de los conjuntos que no son miembros de sí mismos, no es un miembro de sí mismo. Ahora bien, si no es un miembro de sí mismo, entonces debe estar incluido en la lista de los conjuntos que no son miembros de sí mismos y, por lo tanto, pertenece a sí mismo. Ambas alternativas conducen, por lo tanto, a contradicciones. Eso es una paradoja. Más 
específicamente, ésta es la paradoja de Russell. Nótese que este, a primera vista, inofensivo o inocente juego lingüístico hirió letalmente al sistema de Frege, es decir, el primer intento por proveer una fundamentación real y segura para las matemáticas.

No es mi propósito entrar aquí en intrincados vericuetos de teoría de conjuntos y de lógica y, afortunadamente, para nuestros objetivos no es necesario hacerlo. Una razón es que ya en el lenguaje natural nos enfrentamos a lo que básicamente es el mismo problema. Considérese el siguiente caso: un francés afirma: 'Todos los franceses son mentirosos'. Lo que dice es verdadero si y sólo si lo que dice es falso, y a la inversa. Esto es importante porque muestra que el problema (si lo es realmente) estaba ya allí mucho antes de que los lógicos lo inventaran o crearan o plantearan. Esto a su vez sugiere o más bien indica que es un error pensar que porque se puede formalizar el razonamiento y "resolver" el problema en los cálculos, en los lenguajes formalizados, automáticamente resolvimos nuestro problema en todo otro contexto simbólico. Pero sí podría pensarse que si, estrictamente hablando, el problema no pertenece a la lógica, entonces en lugar de estipulaciones, demostraciones y teorías lo que se requiere es, como Wittgenstein insistirá, análisis y elucidación. No es, por lo tanto, de la lógica de la que se debería esperar la resolución total y definitiva del enigma.

\section{El enfoque de Russell y su solución}

La solución russelliana de la paradoja es la famosa teoría de los tipos lógicos. Esbocemos la posición de Russell. De acuerdo con él, las paradojas surgen debido a un cierto "círculo vicioso", el cual puede ser descrito en por lo menos dos formas o "lenguajes", viz., el lenguaje de las clases y el de las funciones proposicionales. Podemos, en efecto, decir que el círculo vicioso se crea por admitir como legítimas totalidades que se incluyen a sí mismas como uno más de sus miembros o, alternativamente, podemos decir que se deriva de la aceptación de la idea de que hay funciones proposicionales que pueden ser sus propios argumentos.

El principio del círculo vicioso enuncia que esta forma de expresar algo no puede tolerarse y, por lo tanto, que las expresiones que no se ajusten a él deben de ser consideradas absurdas o asignificativas. Este principio da de inmediato lugar o de hecho equivale al reconocimiento de una jerarquía de niveles dentro del lenguaje y, en verdad, a una jerarquía de lenguajes (puesto que de acuerdo con él el significado resultará ser "sistemáticamente ambiguo"). Ciertamente podemos hablar de totalidades, pero entonces nos hallamos en un nivel diferente al de ellas (superior) y no puede afirmarse de lo que 
decimos que es parte de esa totalidad. Esto tiene consecuencias algo extrañas. Supóngase que afirmo que todas las proposiciones son verdaderas o falsas. Se sigue, por el principio de Russell, que no puede considerarse lo que digo como una proposición "del mismo nivel". Él, desde luego, añadiría de inmediato "en el mismo sentido", pero esto sólo aumenta nuestros problemas porque:

1) Se sigue que lo que digo no es ni verdadero ni falso en el sentido en que las proposiciones de las que hablo lo son.

2) Sugiere que poseemos una variedad infinita de nociones de verdad y falsedad, correspondientes a los diferentes niveles de lenguaje.

3) La noción misma de proposición se vuelve extremadamente oscura porque ¿en qué sentido es lo que dije diferente de las proposiciones usuales? No es en lo más mínimo obvio que sean diferentes en un sentido esencial y si no lo son ¿cuál es la relevancia de sus respectivos "tipos lógicos" para su significación?

Regresaré sobre estas dificultades más adelante, pero ahora quisiera continuar con la exposición informal de la teoría de Russell.

La solución de Russell al problema de las paradojas consiste en bloquear, mediante una prescripción, la formación de expresiones "ilegítimas" y en declararlas absurdas. Él identifica "tipo" con "rango de significación". Dos expresiones son del mismo tipo si pueden reemplazarse mutuamente sin que dicho reemplazo nos lleve de expresiones significativas a absurdos, tal y como éstos quedaron caracterizados por el principio del círculo vicioso. Éste parece ser un procedimiento perfectamente legítimo al construir un cálculo, puesto que podemos elaborar el cálculo que queramos como queramos, es decir, de acuerdo con las reglas que nosotros escojamos o fijemos, pero no parece que se pueda proceder de igual modo en relación con el lenguaje natural. En todo caso, parece seguirse que la propuesta de Russell, en última instancia, consiste en que, siempre que nos veamos frente a una paradoja, formalicemos las expresiones involucradas y apliquemos el principio del círculo vicioso. Si detectamos una anomalía, simplemente expulsamos la expresión (oración) problemática del reino de las oraciones significativas.

Consideremos entonces nuestro caso problemático y veamos cómo funciona la teoría. Supóngase que afirmo 'Yo siempre miento'. Si lo que digo es verdadero, entonces lo que digo es falso y al revés. Ahora bien, de acuerdo con Russell, esto no es una situación aceptable. Debe haber un error en alguna parte y él piensa que su teoría tanto lo detecta como lo erradica. La explicación corre más o menos como sigue: lo que sucede es que en el momento $t_{1}$ yo afirmo una proposición $P_{1}$, la cual asevera que, en cualquier otro tiempo $t_{\mathrm{n}}$, 
para $t_{1} \neq t_{\mathrm{n}}$, toda proposición que yo afirme será falsa. Evidentemente, $P_{1}$ no pertenece a esa totalidad. Aquí la duda que de inmediato nos asalta es: ¿es lo que yo digo una proposición o no? ¿Se le entiende, es traducible a otros idiomas o no? ¿Vamos simplemente a estipular que no es una proposición genuina porque nos crea problemas? Charles Chihara ha ofrecido una buena reconstrucción del problema en referencia a Epiménides el cretense, quien dijo que todos los cretenses eran mentirosos. Chihara hace ver que "Epiménides asevera una única proposición $q$ en el momento $t$, en donde $q$ es la proposición de que hay una proposición aseverada por Epiménides en $t$ que es falsa. Puesto que $q$ es la única proposición aseverada por Epiménides en $t$, podemos concluir que para toda proposición $p$, si Epiménides asevera $p$ en $t$, entonces $p=q$. Se supone que a partir de estas premisas concluimos que $q$ es verdadera si y sólo si $q$ es falsa. El razonamiento procede esencialmente como sigue.

Sustitúyase [A $p$ ] por [Epiménides afirma $p$ en el tiempo $t_{0}$ ] y sustitúyase $[\mathrm{V} p]$ por [ $p$ es verdadera]. Podemos entonces aseverar, como verdaderas, tanto 'A $q$ ' como ' $(p)$ (A $p \rightarrow p=q)$ ', en donde $q$ es la proposición (expresada por) ' $(\exists p)$ (A $p \& \sim \mathrm{V} p)$ '. Se sigue que si $\mathrm{V} q$, entonces $(\exists p)(\mathrm{A} p \& \sim \mathrm{V} p)$. De ahí que concluyamos que $\sim \mathrm{V} q$. Por otra parte, si $\sim \mathrm{V} q$, entonces $(p)(\mathrm{A} p \rightarrow \mathrm{V} p) \mathrm{y}$, por ello, $\mathrm{V} q$. Por lo que $\mathrm{V} q$ si y sólo $\mathrm{si} \sim \mathrm{Vq} .^{1}$

Debería observarse que la validez del razonamiento se funda en el supuesto de que hay algo así como una totalidad de proposiciones. Pero esto es precisamente lo que Russell rechaza. Por lo tanto, no podemos realmente decir cosas como ' $(p)(\mathrm{A} p \rightarrow p=q)$ ', porque aquí ' $p$ ' y ' $q$ ' no están al mismo nivel. Es así como se nos muestra que la paradoja surge debido a un error lógico.

Antes de examinar críticamente la solución de Russell, intentemos poner en claro cuáles son sus presuposiciones. Las más conspicuas son, me parece, las siguientes:

1) El lenguaje natural es un cálculo, si bien uno altamente deficiente;

2) el lenguaje natural está regido por la lógica;

3) no hay diferencia esencial entre las paradojas lógicas y matemáticas, por una parte, y las "paradojas del lenguaje natural", por la otra;

4) una solución "técnica" puede igualmente funcionar fuera de los lenguajes formalizados o relativamente formalizados;

${ }^{1}$ Charles Chihara, Ontology and the Vicious-Circle Principle. Ithaca/Londres, Universidad de Cornell, 1973, p. 8. 
5) la lógica proporciona el punto de vista de Dios y es mediante ella que podemos describir de manera totalmente neutral cualquier situación y dirimir cualquier problema que surja en el lenguaje.

Una vez dicho esto, podemos pasar ahora a considerar rápidamente el rechazo de Wittgenstein a la solución russelliana al problema de las paradojas, tal y como lo enuncia en el Tractatus.

\section{La solución de Russell y el Tractatus}

En el Tractatus, Wittgenstein eleva toda una gama de objeciones en contra del atomismo lógico de Russell y ofrece una muy variada crítica de la teoría de los tipos lógicos (por ejemplo, de los axiomas de reducibilidad y de infinitud), pero aquí quisiera concentrarme en una objeción concreta. Ésta es simple pero, en caso de ser válida, destruye toda la aportación, dolorosamente erigida, de Russell. Muy a grandes rasgos, dicha objeción consiste en sostener que Russell peca en contra de su propia teoría o, más exactamente, que la teoría misma no cumple con las condiciones que le impone a cualquier discurso o lenguaje, de la clase que sea, para que se le pueda considerar como significativo. Como siempre en el Tractatus, el pensamiento de Wittgenstein es trasmitido por medio de unas cuantas palabras, como si no hubiera absolutamente nada más qué decir sobre el asunto: "el error de Russell", nos dice, "se manifiesta en que, para establecer las reglas de los signos, tuvo que hablar de sus significados". ${ }^{2}$ Una vez más, como en todas partes en el Tractatus, lo que Wittgenstein establece es, una vez que se entiende, claro y contundente, sólo que la condición es hacer explícitos los pensamientos involucrados o tácitos de su razonamiento. En este caso, a lo que Wittgenstein está apuntando es ni más ni menos que a una contradicción por parte de Russell, consistente en usar uno y el mismo lenguaje para referirse a entidades de diferentes tipos. Puesto que no hay manera de enunciar lo que Russell quiere salvo haciéndolo en el lenguaje natural, parecería que no es posible que ninguna teoría al respecto podría siquiera formularse. Si esto es correcto, entonces, obviamente, la solución de Russell se derrumba.

Curiosamente, sin embargo, el punto de vista de Wittgenstein en el Tractatus es sorprendentemente similar al de Russell, lo cual es comprensible

${ }^{2}$ Ludwig Wittgenstein, Tractatus Logico-Philosophicus. Londres, Routledge and Kegan Paul, 1978, 3.33. En vista de lo inaceptable de multitud de proposiciones en las traducciones al español, recurro a mi versión del texto, versión completa que espero algún día publicar. 
puesto que ambos se inscriben en una misma corriente de pensamiento. Wittgenstein admite que lo que Russell trata de decir y de hacer es correcto, pero objeta que no se puede decir. En esta etapa de su pensamiento él todavía admite que las paradojas en efecto plantean un problema real, el cual exige y está en espera de una solución. No obstante, sostiene también que no es mediante una teoría que ello podría lograrse. La idea común queda expresada como sigue (acompañada, como veremos, de un desdeñoso y un tanto injusto comentario por parte de Wittgenstein): "Ninguna proposición puede enunciar nada acerca de sí misma, puesto que el signo proposicional no puede estar contenido en sí mismo (eso es toda la "teoría de los tipos"). ${ }^{3}$ La rectificación frente a la posición de Russell consiste simplemente en hacer ver que la teoría de los tipos tiene que ser reemplazada por una enunciación de las reglas lógicas elementales de todo simbolismo correcto, una formulación que muestre de manera "perspicua" la estructura jerarquizada del lenguaje. Ésta, al ser aprehendida o comprendida, de manera natural nos haría dejar de construir expresiones y fórmulas aparentemente inocentes, pero en el fondo profundamente dañinas, ilegítimas y absurdas. Lo que se requiere para evitar las paradojas es tener presente (ante la mente, si se quiere) las reglas de la sintaxis lógica. La relevante para nosotros es la siguiente: "Una función no puede ser su propio argumento, puesto que el signo de función contiene ya el prototipo de su propio argumento y no puede contenerse a sí mismo". ${ }^{4}$ Así, si ya entendimos, por ejemplo, que ' $f a$ ' es una fórmula bien formada, habremos comprendido que ' $a$ ' puede ser un argumento para ' $f$ ', que ' $a f$ ' no puede ser una fórmula bien formada, que ' $a$ ' es un argumento posible de ' $f$ ' pero que la inversa no puede ser ni tiene sentido tratar de expresarla, y así sucesivamente. Una vez que hemos aprehendido los mecanismos lógicos de nuestros símbolos, ya no estaremos tentados de recurrir a expresiones de la forma ' $f(f)$ ', esquema típico de las fórmulas paradójicas. Pero es de primera importancia no olvidarse de que nada de esto puede enunciarse: hay que "verlo" en el funcionamiento de nuestra notación. Es así como se torna imposible que surjan paradojas.

Esto puede parecer una posición atractiva, pero por lo menos dos argumentos pueden formularse en contra de ella. El primero es que, si funciona, funciona ante todo para lenguajes formales. Pero, más importante aún, se puede objetar que la doctrina lleva a Wittgenstein a una situación igualmente paradójica y puede usarse en su contra su propio argumento. Porque Wittgenstein también, como Russell se lo señala en su "Introducción" al Tractatus, se

${ }^{3}$ Ibid., 3.332.

${ }^{4}$ Ibid., 3.333a. 
las arregla para decir toda una serie de cosas que no pueden decirse (de hecho, toda una concepción del lenguaje, un cierto cuadro de la realidad, etcétera) y que su propia doctrina explícitamente prohíbe. Esto es innegable: acabamos de citar una regla lingüística que pertenece a un conjunto de reglas que, según el mismo Wittgenstein, no pueden enunciarse. Por ello, Wittgenstein tiene que admitir al final de su libro que todo lo que él ha escrito es absurdo. "Mis proposiciones son elucidatorias de este modo: quien me comprende termina por reconocer que son sinsentidos, si las uso para, a través de ellas, salir de ellas. (Por así decirlo, tiene que tirar la escalera después de haber subido por ella)" 5 Pero aquí se plantea un problema serio, porque ¿cómo podría un conjunto de sinsentidos echar por tierra una teoría lógica y además una teoría que, aunque con implicaciones indeseables, de hecho funciona, puesto que la teoría de Russell logra efectivamente evitar las paradojas (por lo menos las lógicas)? Parecería, pues, que nos encontramos en la harto rara situación de estar obligados a escoger entre una teoría que incurre en una petición de principio y una doctrina que lleva a sinsentidos. Ciertamente ambas posiciones contienen intuiciones valiosas e importantes, pero no puede admitirse que, así como están, hayan "resuelto" el problema de las paradojas. Ahora bien, parecería que, independientemente de su coherencia interna, hay otra razón que nos impide ver en cualquiera de ellas la solución real del problema, a saber, que ni en el Tractatus ni en la obra de Russell hay un esfuerzo por comprender el problema. Russell le teme a las contradicciones y da por supuesto que se trata de una clase peligrosa y dañina de entidades. Para Wittgenstein las contradicciones no son propiamente hablando proposiciones, si bien junto con las tautologías contribuyen a fijar los límites de la significatividad de un lenguaje dado. En relación con las paradojas de la lógica y la teoría de conjuntos, la posición de Wittgenstein, sin embargo, comporta una notable intuición: él sugiere que las contradicciones surgen porque hay algo que no percibimos, que no detectamos debidamente. Éste es el eslabón que nos lleva directamente a la obra posterior de Wittgenstein. En ella, es la concepción usual (logicista) misma de las contradicciones y de los inmensos problemas que éstas supuestamente plantean lo que se rechaza. Desde esta perspectiva, lo que necesitamos no es una teoría más, un cálculo más, nuevas pruebas o demostraciones, reglas informulables. Lo que se requiere es más bien análisis conceptual, porque todo el problema surge debido a una confusión: se trataría, desde esta nueva perspectiva, de un típico enredo filosófico, de un seudoproblema.

${ }^{5}$ Ibid., 6.54a. 


\section{Notas aclaratorias del Wittgenstein maduro}

Una primera idea importante del Wittgenstein de la madurez en relación con el problema de las paradojas es que una contradicción debe de ser algo inesperado, una mala sorpresa y algo que efectivamente nos molesta, obstaculiza o perturba. Esta sencilla idea tiene dos consecuencias muy importantes. Por ejemplo, entre otras cosas implica que:

a) Una contradicción oculta o latente pero que, sin embargo, nunca se manifiesta no es una contradicción. "Una contradicción es una contradicción sólo si está allí”, ${ }^{6}$ es decir, si (querámoslo o no) efectivamente aparece.

b) Una contradicción genuina no es algo creado artificialmente.

Consideremos ambas cuestiones en ese orden.

Creo que podemos de inmediato afirmar que nuestro problema se divide en dos sub-cuestiones, una concerniente al lenguaje y otra referente a la lógica. De hecho, en la vida y el lenguaje ordinarios, nosotros no nos preocupamos por las contradicciones. Si alguien viene y nos dice 'Está lloviendo y no está lloviendo' no intentaremos hacerle ver que está en un error. Más bien, nuestra respuesta será algo como: 'Por favor, sé un poco más serio' o ‘Estás tratando de burlarte de mí?' o 'Deja de decir tonterías', etcétera, o simplemente no le prestamos atención a lo que dice. Podría de inmediato objetarse que es obvio que no se puede adoptar la misma actitud en lógica o en matemáticas. Pero ¿por qué no? Para responder a esta pregunta, tenemos que distinguir dos clases de contradicciones: o bien inesperadamente nos topamos con una contradicción o bien nosotros mismos la construimos. Ahora bien, si nos encontramos frente a una contradicción de nuevo nos hallamos frente a dos posibilidades:

1) Se trata de la contradicción (i. e., no hay más que una), o

2) es tan sólo una de un número indefinido de ellas.

Nuestra actitud será diferente en cada caso (o debería serlo). En el primero, Wittgenstein sugiere, lejos de temerle, deberíamos estar contentos por haberla encontrado. Sería como haber descubierto los restos de un animal hasta ahora desconocido, una rareza. Por lo que el caso peligroso realmente

${ }^{6}$ B. F. McGuinness, ed., Ludwig Wittgenstein and the Vienna Circle. Oxford, Basil Blackwell, 1979, p. 120. 
parece ser el segundo. Regresemos entonces a nuestra dicotomía anterior. Hay montones de contradicciones que no podemos evitar y hay montones de contradicciones que nosotros construimos. Lo que Wittgenstein parece mantener es que la segunda posibilidad no tiene ningún interés: si la existencia de las contradicciones, que tanto tememos, depende de nosotros, entonces no hay problema: sencillamente dejemos de construirlas y asunto arreglado. "Eso es lo que resulta por construir tales oraciones. -¡Pero hay una contradicción aquí! -Bueno entonces hay una contradicción aquí. ¿Hace acaso algún daño?”7 Todo esto, sin embargo, hace ver que el problema real se plantea cuando nos enfrentamos con el fenómeno de una multiplicidad de contradicciones que son inevitables. La pregunta a la que hay que responder es, por lo tanto, la siguiente: ¿cuándo surge ese problema?

Una vez más, hay dos modos como las contradicciones que nos incumben podrían generarse. Podemos empezar con proposiciones contradictorias o podemos tener reglas de inferencia inconsistentes. Parecería evidente, en efecto, que pares de proposiciones contradictorias pueden aparecer ya sea al principio ya sea durante la construcción o el desarrollo del cálculo. Wittgenstein sostiene que la primera "posibilidad" sencillamente no tiene sentido. Su argumento es similar a lo que sería parte de su respuesta al escéptico filosófico y probablemente sería algo como esto: "Mira, para que afirmes o niegues lo que afirmas o niegas, necesitas usar el lenguaje tal como es. Ahora bien, la significatividad de nuestro lenguaje presupone precisamente lo que tú niegas o, si afirmas algo, en él (i. e., el juego de lenguaje) no hay lugar para tus propias asunciones". Ilustremos esto con el caso del ajedrez. Sencillamente no hay posiciones contradictorias al comienzo; no tiene sentido afirmar tal cosa. Si hubiera posiciones contradictorias al inicio del juego no habría juego; éste no sería posible. De ahí que un argumento viable corra como sigue: para que las contradicciones hagan su aparición algún juego debe ya jugarse. Es sólo porque hay un punto de partida fijo, libre de contradicciones, que tiene sentido hablar posteriormente de contradicciones. Pero es claro que en el punto de partida no puede haberlas, $i$. e., no tiene sentido decir que las hay. En palabras de Wittgenstein:

Los axiomas tienen dos significados, como vio Frege:

1) Las reglas de acuerdo con las cuales se juega.

2) Las posiciones de apertura del juego.

${ }^{7}$ L. Wittgenstein, Remarks on the Foundations of Mathematics. Londres/Cambridge, MIT, 1975, p. 51, sec.11. 
Si se toma a los axiomas en el segundo sentido, no puedo concederle ningún sentido a la afirmación de que se contradicen entre sí. ${ }^{8}$

Nadie teme una contradicción en esta etapa, porque no habría nada más fácil que suprimirla o cancelarla o eliminarla: sencillamente extraemos una de las oraciones "contradictorias". No es, por lo tanto, esta clase de contradicción la que preocupa a los lógicos.

Llegamos, así, al verdadero problema de las contradicciones en los sistemas formalizados. El problema consiste en que éstos pueden incluir reglas que, de maneras no obvias, generen contradicciones. Parecería que, en la medida en que no hayamos demostrado la consistencia de nuestros sistemas no podremos estar seguros de ellos; por consiguiente no podrán ser considerados como significativos y, por ende, no podrán ser empleados. Sería por eso que necesitamos urgentemente pruebas de consistencia; sería gracias a dichas pruebas que demostraríamos que ninguna de las reglas usadas en el sistema de hecho es susceptible de llevarnos de verdad a falsedad y, por lo tanto, a contradicciones. Pero ¿cómo llevamos a cabo tales pruebas? Parece haber sólo dos formas:

a) O bien argumentamos en el meta-lenguaje.

b) O bien usamos las mismas reglas o reglas equivalentes para efectuar nuestra meta-prueba.

Ahora bien, si esto es así, entonces a todas luces ninguna de las posibilidades permite llegar a lo que se quería. Porque en el primer caso, no hay una prueba formal (la "prueba" estaría dada en prosa, como diría Wittgenstein) y, en el segundo, tendremos siempre el derecho de pedir la prueba de la prueba, la prueba de la prueba de la prueba y así ad infinitum.

Ahora bien, la primera clase de prueba de consistencia representa, por decirlo de alguna manera, intuitivamente, un esfuerzo por eliminar posibles fuentes de incomprensión mediante la explicación, la aclaración de ciertas cosas. Esto desde luego no basta, pero apunta en la dirección correcta. Wittgenstein expone la idea como sigue:

Ahora bien, debe decirse que estas antinomias no tienen nada que ver con las contradicciones de las matemáticas, que no hay aquí ninguna conexión. Porque las antinomias no surgieron en el cálculo, sino en el lenguaje usual, y ello precisamente porque se usan las palabras de

${ }^{8}$ B. F. McGuinness, ed., op. cit., p. 119. 
manera ambigua. [...] Las antinomias se disuelven gracias a un análisis, no por medio de una prueba.

Si las contradicciones en matemáticas surgen por una falta de claridad, entonces no podré nunca despejar la falta de claridad por medio de una prueba $[\ldots]$

Esto ya muestra que no se puede dar tal una prueba de consistencia [...], que la prueba no puede dar lo que se espera de ella. ${ }^{9}$

Para comprender y apreciar lo que Wittgenstein está diciendo es menester hacer ciertas aclaraciones.

Es claro que el ataque de Wittgenstein se desarrolla simultáneamente en tres direcciones diferentes. Lo primero que hace es rechazar la identidad de la lógica con las matemáticas. Se sigue que una contradicción o una antinomia en lógica no puede afectar la consistencia de las matemáticas. Por otra parte, puesto que Wittgenstein desprovee a la lógica de su realidad etérea, divina, "ultrafísica", etcétera, resulta que lo más que ésta puede hacer es reflejar de manera idealizada ciertos aspectos o mecanismos de nuestro lenguaje. Esto confirma el punto de vista de Wittgenstein de que el problema de las contradicciones surge, en primer término, en el lenguaje natural. La aparición de las contradicciones y el malestar que provocan (en lógica sobre todo) nos obligan a re-pensar las relaciones que se dan entre el lenguaje, la lógica y la praxis, es decir, entre la lógica y su aplicación, por una parte, y el lenguaje y la acción, por la otra. Desde un punto de vista meramente sintáctico, una contradicción no es más alarmante que una tautología o que una proposición muy compleja. A final de cuentas no es sino una fórmula más. El problema, por lo tanto, surge con su interpretación, la cual significa con la línea de acción con la que podría estar asociada. Una vez visto esto, entonces sí estamos en posición de comprender por qué las contradicciones representan un problema: lo que sucede con ellas es que nos confunden, nos paralizan. Lo que ellas impiden que tenga lugar es la acción. Esto amerita un mínimo de aclaración.

Es la fructífera idea de Wittgenstein de ver en las palabras utensilios, lo que funciona como plataforma básica para el desarrollo de una nueva forma de entender el problema general de las contradicciones y las paradojas. Esta perspectiva, por ejemplo, nos capacita para dejar de hablar de proposiciones como si fueran entidades de alguna extraña clase y orienta nuestra atención hacia los signos, hacia las oraciones. En esta nueva concepción el papel que jugaban las antiguas proposiciones lo desempeñan ahora los movimientos en

${ }^{9}$ L. Wittgenstein, Observaciones filosóficas. Trad. de Alejandro Tomasini Bassols. México, UNAM, Instituto de Investigaciones Filosóficas, 1997, p. 307. 
los diversos juegos del lenguaje. Podemos ahora parafrasear el problema que plantean las contradicciones: lo que sucede es que lo que normalmente está prohibido hacer es usar simultáneamente dos utensilios, en este caso $p$ y $\sim p$, diseñados para indicar cursos de acción que se excluyen o cancelan mutuamente. Podríamos presentar la idea más o menos como sigue: pretender usarlos al mismo tiempo equivale en realidad a jugar un juego al que podríamos llamar 'el juego en el que todo está permitido' y el problema es que con ese juego lo que de hecho se hace es abolir todos los juegos. Es por eso que no es legítimo usar simultáneamente los instrumentos " $p$ " y " $\sim p$ ". Pretender servirse al mismo tiempo de dos instrumentos lingüísticos como esos sería como estar haciendo trampa. Ahora bien, el hecho de que en una situación determinada optemos por el instrumento " $p$ " no implica que hayamos excluido o desterrado para siempre el utensilio " $\sim p$ ": significa simplemente que en esa ocasión particular no estamos autorizados para emplearlo o que prohibimos hacerlo. Una condición necesaria (mas no suficiente) para que nuestro pensamiento sea operativo y efectivo es precisamente que sea lo que llamamos "coherente", por lo que de manera natural cada vez que teorizamos, conversamos, especulamos, calculamos, etcétera, optamos por uno u otro de los instrumentos de los que permanentemente disponemos (oraciones), sin por ello (repito) sentirnos compelidos a desprendernos de uno de los dos para siempre. Un utensilio que no sirve en una ocasión puede servir perfectamente bien en otra. Así, por ejemplo, puedo tener que optar entre el pensamiento de que llueve y el pensamiento de que no llueve, entre otras razones para decidir si salgo con paraguas o no. Supongamos que, dada la estación, opto por el instrumento 'llueve'; dicha elección de ninguna manera implica que nunca más podré apelar al instrumento 'no llueve'. El problema con 'llueve y no llueve' es que no me permite actuar. Y es para indicar nuestro interés en salvaguardar esa utilidad alternativa de los instrumentos lingüísticos que son las oraciones que tenemos una ley lógica como el principio de no contradicción $\sim(p \& \sim p)$. En otras palabras, la lógica hace ver qué aceptamos como inferencia válida dado un conjunto de oraciones, enunciados, proposiciones o creencias, pero es un hecho que nosotros no evitamos las paradojas o caer en contradicciones por no tener conflictos con la lógica. Lo que quiero sugerir es simplemente que hay un sentido interesante en el que hay algo más fundamental, "anterior" o "previo" a la lógica misma, que es lo que realmente nos importa y que tiene su reflejo en ella. Veamos rápidamente de qué se trata.

Desde el punto de vista de la consistencia lógica, sabemos que el lenguaje natural es "deficiente"; contiene, por ejemplo, la paradoja del mentiroso. Pero si ello es así ¿no es acaso sorprendente que no estemos continuamente extrayendo en él cualquier conclusión que deseemos? ¿Por qué nadie razona de manera lógicamente correcta como sigue: "Todos los mexicanos son menti- 
rosos; yo soy mexicano y, por lo tanto, la luna es de queso"? La respuesta más simple parece ser que, cuando nos las habemos con contradicciones, simplemente las ignoramos. Así procedemos porque el efecto inmediato de las contradicciones y las paradojas es paralizarnos, lingüística y extra-lingüísticamente. Llegar a una contradicción es como haber desembocado en un callejón sin salida: hay que echar marcha atrás y buscar otro camino. En general, los usuarios queremos usar el lenguaje para actuar (en un sentido amplio de la expresión). Es en conexión con las prácticas (las "formas de vida") que éste existe. El problema con las contradicciones y las paradojas es, por así decirlo, que hipnotizan al lenguaje, impidiéndole funcionar de modo que las prácticas humanas resultan afectadas. Lo que con las contradicciones se logra es simplemente abrir la puerta para que reine el caos en el lenguaje, con lo cual potencialmente se le inutiliza. La emisión y admisión de contradicciones es simplemente la manifestación formal o simbólica de una incomprensión respecto a lo que es el uso apropiado del lenguaje. Lo que significa que una contradicción haga su aparición es que la maquinaria de signos y reglas que es el lenguaje está trabada. Empero, y este es el punto importante, el remedio para dicha enfermedad no proviene de la lógica misma, que a lo sumo sólo puede mostrar, exhibir y diagnosticar el mal. El problema no tiene sus raíces en la lógica. De hecho hasta podría decirse que si las contradicciones cumplieran alguna función socialmente reconocida (lúdica o de entretenimiento, por ejemplo) serían bienvenidas. De ahí que lo que realmente es importante para nosotros sea la superación del fenómeno de parálisis o de falta de acción y éste no es tanto un asunto de lógica como de comprensión de que se está pretendiendo hacer un uso inapropiado del instrumental lingüístico del que se dispone.

Hay una clase fundamental de conflictos que queda recogida en el simbolismo formal, en la lógica, bajo la forma de contradicciones. En otras palabras, hay ciertos estados de los hablantes asociados con el juego formal de las contradicciones que son lo que realmente nos interesa evitar, eludir, sortear, a saber, estados como los de duda, titubeo, vacilación, etcétera. No deberíamos olvidar, desde luego, que hay tanto dudas reales como seudodudas (e. g., dudas filosóficas) y que en ambos casos se aplican los verbos ordinarios ('no estoy seguro de que...', 'dudo que...', etcétera). La diferencia primordial entre esas clases de dudas es que toda duda genuina se refleja en la acción o, mejor dicho, en la no acción. Así como cuando alguien duda no sabe qué hacer, así también cuando alguien es contradictorio tampoco sabe realmente qué hacer, puesto que puede hacer absolutamente cualquier cosa. Esto sugiere que hay una conexión interesante entre el estado de duda real y las contradicciones lógicas: parecería como si las contradicciones no fueran otra cosa que la expresión formal de estados malsanos (anti-prácticos, por así llamar- 
los) indicados por palabras como "vacilar", "excluir", "dudar" y así sucesivamente. En todo caso, el punto es que la razón por la que por todos los medios tratamos de evitar contradicciones es que éstas representan simbólicamente algo que es lo que realmente tememos y tratamos de evitar, esto es, aquellos estados o situaciones en los que no sabemos qué hacer. Es por eso a final de cuentas que las contradicciones son tan "temibles". Intentemos aplicar a la lógica la idea que está aquí contenida.

El problema puede ser planteado de manera muy sencilla como sigue: ¿por qué no se procede en lógica al modo como procedemos en el lenguaje natural, esto es, por qué un lógico no se abstiene de extraer deliberadamente conclusiones cuando se encuentra frente a dos utensilios que se contraponen? ¿Sería acaso imposible que se produjera la siguiente situación: tenemos un lenguaje formalizado, efectuamos nuestras inferencias, notamos que ciertas asunciones llevan a contradicciones y deliberadamente las evitamos? Es ésta una idea con la que Wittgenstein parece jugar: "Lo que quiero decir es, pues, esto: si surgieran contradicciones en las reglas del juego de las matemáticas, lo más fácil del mundo sería remediarlo. Lo único que tenemos que hacer es una nueva estipulación que cubra el caso en el que las reglas entran en conflicto y asunto arreglado". ${ }^{10}$ De ahí que si a lo que le tenemos pavor es a las contradicciones ecuál es el problema con introducir una ley como: "Siempre que llegues a una fórmula como ésta simplemente ignórala y empieza de nuevo tu razonamiento con un conjunto modificado de premisas"? ¿Qué se podría objetar a quien dijera: "Siempre que tengas en tu sistema utensilios (oraciones) que se excluyen mutuamente, trabaja con el que realmente te sirve y deja de jugar simultáneamente con los dos"? En verdad ¿por qué no sería esto, o algo parecido (podríamos imaginar, por ejemplo, una formulación "técnica" de ello), una solución al problema de las paradojas?

Un error que he tratado de evitar aquí es el de los lógicos en general, muy bien ejemplificado por Chihara. ${ }^{11} \mathrm{Al}$ discutir la solución de Wittgenstein, él se queja de que este último no ofrece ninguna solución "técnica" y, por consiguiente, infiere que Wittgenstein sencillamente no vio el problema el cual, si no nos hemos equivocado, con lo que tiene que ver es más con la aplicación de la lógica que con la lógica. Por mi parte, estoy convencido de que, al contrario, es Chihara quien no comprendió el nuevo enfoque del problema. Des-

${ }^{10}$ Ibid., p. 306.

${ }^{11}$ Véase, por ejemplo, sus artículos "Mathematical Discovery and Concept Formation" y "Wittgenstein and Logical Complusion", en G. Pitcher, ed., Wittgenstein. The Philosophical Investigations. Londres, Macmillan, 1970, y "Wittgenstein's Analysis of the Paradoxes in his 1939 Lectures on the Foundations of Mathematics", en Philosophical Review, 1977. 
de esta nueva perspectiva, no es un "hecho" interno a la lógica lo que hace tan horribles a las contradicciones, así como tampoco recibimos a las tautologías con el entusiasmo y la intensidad equivalentes a nuestro sentimiento de horror frente a sus hermanas bastardas, $i$. e., las contradicciones. Lo que deberíamos entender es que somos nosotros quienes las dotamos de un carácter "horrible", lo cual se debe a su peculiar relación con el significado, a su carencia de utilidad en el uso normal del lenguaje natural y a nuestro rechazo innato del titubeo y la vacilación. Es precisamente este crucial vínculo lo que de manera superficial intenté describir en este trabajo. 